\title{
Lytic bacteriophage have diverse indirect effects in a synthetic cross- feeding community
}

\author{
Lisa Fazzino ${ }^{1,2}$ • Jeremy Anismann ${ }^{2,3}$ - Jeremy M. Chacón ${ }^{2,4} \cdot$ Richard H. Heineman ${ }^{5}$ - William R. Harcombe $\mathbb{1}^{1,2,4}$
}

Received: 17 July 2019 / Accepted: 15 August 2019 / Published online: 2 October 2019

(c) The Author(s) 2019. This article is published with open access

\begin{abstract}
Bacteriophage shape the composition and function of microbial communities. Yet it remains difficult to predict the effect of phage on microbial interactions. Specifically, little is known about how phage influence mutualisms in networks of crossfeeding bacteria. We mathematically modeled the impacts of phage in a synthetic microbial community in which Escherichia coli and Salmonella enterica exchange essential metabolites. In this model, independent phage attack of either species was sufficient to temporarily inhibit both members of the mutualism; however, the evolution of phage resistance facilitated yields similar to those observed in the absence of phage. In laboratory experiments, attack of $S$. enterica with P22vir phage followed these modeling expectations of delayed community growth with little change in the final yield of bacteria. In contrast, when E. coli was attacked with T7 phage, S. enterica, the nonhost species, reached higher yields compared with no-phage controls. T7 infection increased nonhost yield by releasing consumable cell debris, and by driving evolution of partially resistant E. coli that secreted more carbon. Our results demonstrate that phage can have extensive indirect effects in microbial communities, that the nature of these indirect effects depends on metabolic and evolutionary mechanisms, and that knowing the degree of evolved resistance leads to qualitatively different predictions of bacterial community dynamics in response to phage attack.
\end{abstract}

\section{Introduction}

Bacteriophage significantly influence microbial community structure and function [1]. Phage limit the size of bacterial populations, which can change microbial community composition. For example, phage kill $>20 \%$ of marine bacteria

Supplementary information The online version of this article (https:// doi.org/10.1038/s41396-019-0511-z) contains supplementary material, which is available to authorized users.

William R. Harcombe

harcombe@umn.edu

1 Department of Microbiology and Immunology, University of Minnesota, Minneapolis, MN, USA

2 BioTechnology Institute, University of Minnesota, Minneapolis, MN, USA

3 College of Continuing and Professional Studies, University of Minnesota, Minneapolis, MN, USA

4 Ecology, Evolution, and Behavior, University of Minnesota, Minneapolis, MN, USA

5 Biology Department, Kutztown University, Kutztown, PA, USA every day [2]. Viral infection of bacterial populations not only impacts the composition of bacterial communities, but also influences emergent community functions such as the rate at which nutrients are converted into biomass [3]. As a result, phage critically influence biogeochemical cycling [4], biotechnology [5], the food industry [6], and human health $[7,8]$. Despite the importance of phage in microbial communities, we cannot reliably predict the impact of phage on the composition or function of communities. As we strive to manage microbial communities, we must improve our understanding of responses to phage infection in multi-species systems.

Phage alter competitive bacterial communities by changing the species abundance. When phage kill dominant competitors, weaker competitors that are resistant to phage can flourish, changing species ratios, which can change community function [9-12]. Sometimes, species ratios rapidly revert to pre-phage frequencies once a host evolves phage resistance, but costs of resistance can generate persistent changes in competitive community composition following phage addition [9]. However, phage attack of one species often has little impact on total community biomass. In communities of competitors, a reduction in host biomass 
is typically compensated for by the growth of nonhost competitors [13]. Taken together, in competitive systems, phage alter species ratios, but have little impact on total microbial biomass.

Much less is understood about how phage influence cooperative networks in microbial communities. Microbial communities are often organized into cross-feeding webs in which each species relies on metabolites excreted by others $[14,15]$. Networks of metabolic dependencies have been described in marine, terrestrial, and human-associated microbial communities [14]. While phage are likely present in all of these systems, the impact of phage on the composition and function of cross-feeding microbial communities remains under-studied.

However, the response of cross-feeding communities to abiotic disturbances may inform how cross-feeding communities respond to phage infection. In the absence of disturbance, obligate mutualism typically constrains species ratios such that communities converge on an equilibrium ratio from any initial ratio [16, 17]. This constraint on final species ratios means that limiting one species should indirectly inhibit cross-feeding partners, thereby decreasing total community biomass, but maintaining species ratios [18]. For example, antibiotic treatment of a three-species cross-feeding community that inhibited the most sensitive member inhibited growth of all members of the community by depriving community partners of cross-fed nutrients [19]. Therefore, our null hypothesis is that phage infection on one member of a cooperative network will limit growth of the entire cross-feeding network but will not change species ratios.

Yet, the null hypothesis that phage infection will alter cooperative community biomass but not composition has several underlying assumptions that may not hold. First, it assumes that bacteria obtain nutrients directly from the secretions of bacterial partners. Yet there is a rich body of literature suggesting that phage-mediated cell lysis releases nutrients into the environment. Indeed, this 'viral shunt' is thought to play a major role in global nutrient cycling [20$22]$ and may alter species interactions [23, 24]. Second, the hypothesis overlooks possible ecological consequences of the evolution of phage resistance. For example, it assumes that phage resistance does not alter the exchange of crossfed nutrients. If phage resistance causes changes in either nutrient secretion or uptake, it could alter species ratios, potentially changing community function.

In this study, we sought to determine the effects of phage attack on cooperative communities by combining resourceexplicit mathematical modeling and wet-lab experiments of a synthetic cross-feeding co-culture of Escherichia coli and Salmonella enterica $[17,25]$. An E. coli strain auxotrophic for methionine was paired with a $S$. enterica strain that was evolved to secrete methionine [25]. The pair forms an obligate mutualism in lactose minimal medium, as $S$. enterica cannot consume lactose and instead relies on acetate excreted by $E$. coli during overflow metabolism. Grown under these conditions, these bacteria are a simple two-species cooperative community. To this community, we added either an E. coli-specific (T7) or S. entericaspecific (P22vir) lytic phage and tracked community responses (Fig. 1a). As a null hypothesis, we predicted that cross-feeding would constrain species ratios and therefore targeted phage attack would inhibit growth of the entire community. However, we anticipated that phage resistance would evolve, making biomass reduction temporary. We found that both phage delayed community growth, but neither phage reduced final host yields and T7 infection of $E$. coli led to surprising changes in species ratios.

\section{Results}

\section{Resource-explicit model suggests phage have little impact on final community composition}

We used a resource-explicit model to predict how a cooperatively growing bipartite bacterial community responds to lytic phage attack during batch growth. We modeled densities of E. coli (E), S. enterica (S), and phage (T7 or P22vir), as well as cross-fed metabolite concentrations (Fig. 1a and Supplementary Methods). Growth of each bacterial species was a function of maximum growth rate $\left(\mu_{\mathrm{x}}\right)$ and Michaelis-Menten saturation parameters $\left(\mathrm{k}_{\mathrm{m}}\right)$ of essential metabolites. Bacterial death due to phage infection was modeled as a linear interaction between phage and host, and modified by an adsorption (i.e., predation) constant $(\gamma)$. Phage attack generated new phage particles at a rate set by the burst size $(\beta)$. Phage-resistant hosts $\left(E_{R}\right.$ or $\left.S_{R}\right)$ were initiated at a frequency of $0.1 \%$ in each bacterial population so that resistance alleles increased in frequency during phage infection. The cost of resistance, if any, was represented by a smaller growth rate $\left(\mu_{\mathrm{x}}\right)$ of resistant bacteria. Parameters were informed by the literature and adjusted to match experimental observations in the absence of phage (Supplementary Table 1).

When modeled in the absence of phage, the community converged to $88 \%$ E. coli regardless of the starting bacterial ratio, consistent with previous wet-lab observations (Fig. 1c and Supplementary Fig. 1) [17]. In the absence of phage, sensitive and resistant bacterial genotypes increased in density with relative frequencies determined by the cost of resistance (Supplementary Fig. 2).

The model predicted that the presence of phage increases the time required for the community to reach carrying capacity, but has little impact on the final species yields. Both $\mathrm{T} 7$ and P22vir rapidly killed all sensitive hosts 
A
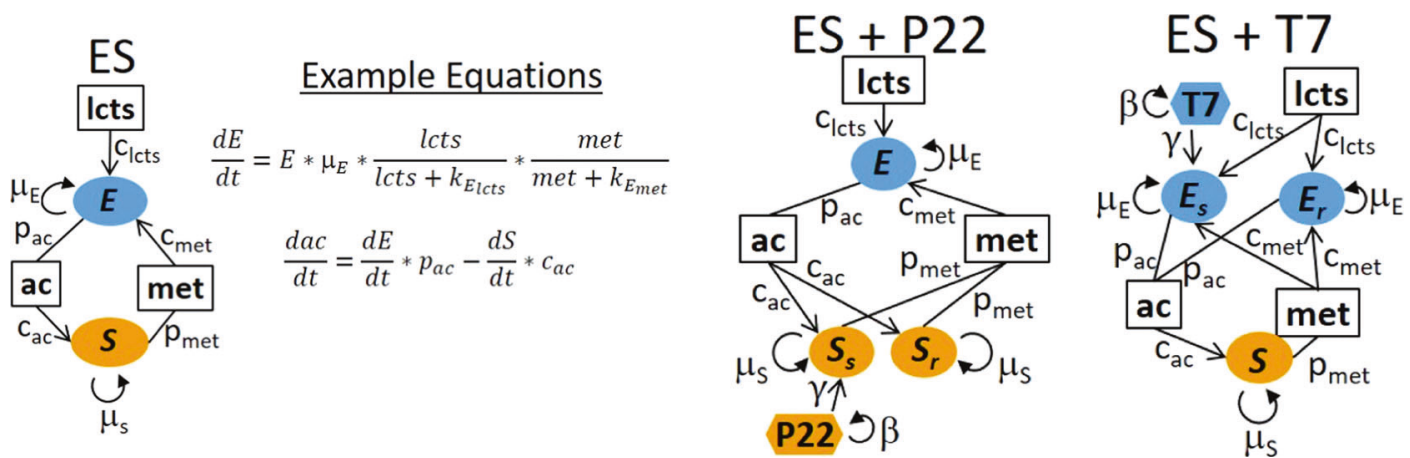

B
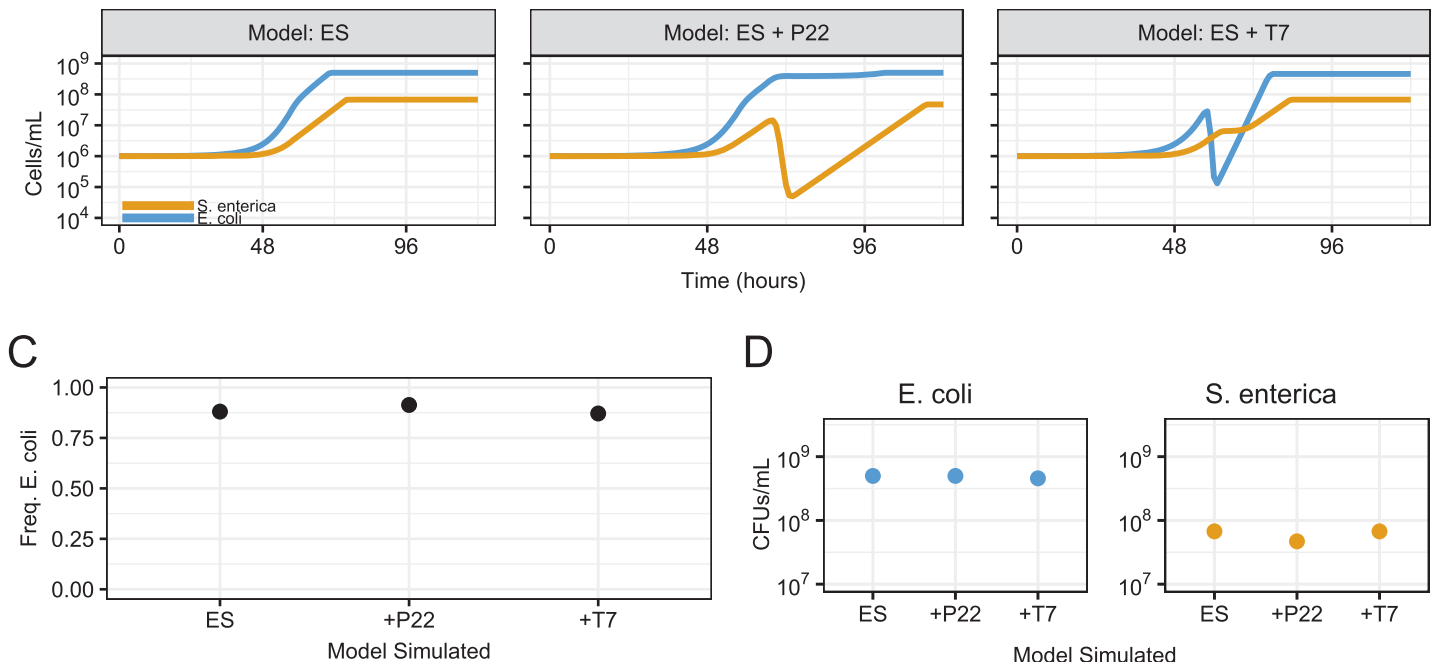

$\mathrm{D}$

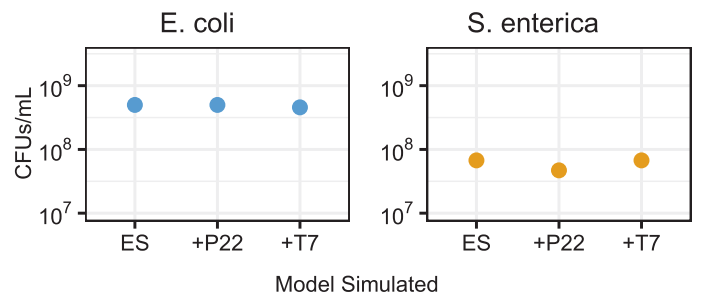

Fig. 1 Mathematical model predicting consequences of phage infection of cooperative co-cultures. a Schematic of models for systems with E. coli (E), S. enterica (S), and phage (T7 or P22). Bacteria are represented by ovals. Phage sensitivity (s) or resistance (r) is indicated by subscripts. Phage are indicated by hexagons and are colored to match their bacterial host. Boxes indicate metabolites (lcts lactose, ac acetate, met methionine). Parameters are next to interaction arrows.

(Fig. 1b and Supplementary Fig. 2). The reduction in the host population reduced the amount of cross-feeding, thereby temporarily stalling community growth. However, phage-resistant hosts rapidly increased in abundance, allowing the community to reach carrying capacity. Host species reached $91 \%$ to $70 \%$ of the final densities as a result of phage attack (Fig. 1d). This reduction is because sensitive host cells consume resources before they are killed by phage, and fewer resources are therefore available for growth of the resistant host. However, sensitive hosts are killed before they consume many resources. No change was observed in the final abundance of the nonhost bacteria. Furthermore, reducing maximum growth rates of phageresistance genotypes as a proxy for the cost of resistance did not change yields, but did cause small delays of community growth (Supplementary Fig. 2a, b). $\mathrm{c}_{\mathrm{x}}=$ consumption rate of subscript nutrient, $\mathrm{p}_{\mathrm{x}}=$ production rate of subscript nutrient, $\mu_{\mathrm{x}}=$ growth rate $\left(\mathrm{h}^{-1}\right), \beta=$ burst size, $\gamma=$ adsorption constant. b Simulated growth curves with and without phage treatment. Yellow $=S$. enterica, blue $=E$. coli. c Species ratios represented with frequency of $E$. coli at time $=125 \mathrm{~h}$. d Final densities of bacteria $($ cells $/ \mathrm{ml})$ in mathematical models at time $=125 \mathrm{~h}$

\section{In wet-lab experiments, $\mathrm{T} 7$ infection changed species ratios while P22vir infection did not}

Using our wet-lab experimental cross-feeding system, we tested the mathematical model prediction that phage attack would delay growth but have little impact on the final community. Communities were started with a multiplicity of infection (MOI) of $\sim 0.01$ (Supplementary Table 2). Any resistant host cells arose via mutation during cooperative community growth; they were not intentionally seeded into the host population. Growth of each bacterial species was tracked with a unique fluorescent marker which could be converted to a species-specific OD (Supplementary Fig. 3) [19]. After growth, co-cultures were plated for $E$. coli and $S$. enterica colony-forming units (CFUs), and $\mathrm{T} 7$ or P22vir plaque-forming units (PFUs). 
When the co-culture was exposed to the $S$. entericaspecific P22vir phage, P22vir rose to high titers (Supplementary Fig. 4), caused evolution of resistance in $S$. enterica (Table 1), and delayed community growth compared with no-phage controls $(p=0.0037$, Fig. 2a). However, compared with no-phage controls, P22vir significantly affected neither the species densities of $E$. coli $(p=0.35$, Fig. 2c) or S. enterica ( $p=0.95$, Fig. $2 c)$, nor the frequency of $E$. coli ( $p=0.51$, Fig. 2b). Overall, P22vir infection of the co-culture delayed growth and did not change final community composition, as predicted by co-culture simulations.

The effects of $\mathrm{T} 7$ phage on the cooperative co-culture differed in some ways from the effects of P22vir phage. Like P22vir, T7 phage rose to high titers (Supplementary Fig. 4), caused evolution of resistance in its host - E. coli (Table 1), and delayed community growth compared with no-phage controls $(p=0.0037$, Fig. 2a). However, there were changes to the cooperative co-culture composition. T7 infection of $E$. coli in a cooperative community decreased E. coli density in the presence of T7 ( $p=0.017$, Fig. 2b). In fact, $E$. coli went extinct in two of the five T7 phage replicate communities. In addition, the final frequency of $E$. coli relative to no-phage controls decreased following $\mathrm{T} 7$ phage attack ( $p=0.008$, Fig. 2c). Surprisingly, growth of $S$. enterica was not constrained by the phage-mediated death of E. coli. Instead, S. enterica reached between 8- and 35-

Table 1 Resistance and mucoidy in phage-treated cooperative cocultures

\begin{tabular}{llll}
\hline Treatment & $\begin{array}{l}\text { No. of replicate } \\
\text { communities }\end{array}$ & $\begin{array}{l}\text { resistant }{ }^{\mathrm{a}} / \text { total } \\
\text { isolates }\end{array}$ & $\begin{array}{l}\text { mucoid/total } \\
\text { isolates }\end{array}$ \\
\hline ES & 10 & $0 / 30$ & $0 / 30$ \\
ES + P22vir & 5 & $15 / 15$ & $0 / 15$ \\
ES + T7 & $3^{\text {b }}$ & $9 / 9$ & $9 / 9$ \\
\hline
\end{tabular}

${ }^{\mathrm{a}}$ Resistance assayed with cross-streaks

${ }^{\mathrm{b}}$ Two replicate communities were driven extinct
A

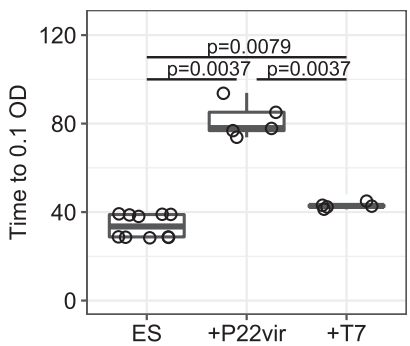

B

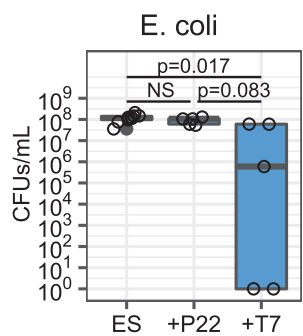

Fig. 2 Experimental data of cooperative co-culture growth and the effect of adding phage. a Time to 0.1 OD for each phage treatment. Statistical significance determined with Mann-Whitney-U test with FDR multiple hypothesis correction. b Boxplot depicting measured $\mathrm{CFUs} / \mathrm{mL}$ of $E$. coli or $S$. enterica at the end of growth. $\mathbf{c}$ Species ratios fold higher density in the presence of $\mathrm{T} 7$ (Fig. 2c). In addition, $S$. enterica entered log-phase sooner, despite its dependence on E. coli secreted acetate (Supplementary Fig. 3a). This led to a rapid increase in biomass of communities with T7 phage (Supplementary Fig. 3b). Phage attack on $E$. coli appeared to release $S$. enterica from the constraints typically imposed on cross-feeding community partners-a novel result that we interrogated further.

\section{T7-resistant $E$. coli increase $S$. enterica densities in the absence of phage}

The increase of $S$. enterica during T7 infection of $E$. coli could result from T7-resistance altering E. coli secretion of cross-fed metabolites. To test if T7-resistant $E$. coli provide more metabolites than sensitive $E$. coli, we assayed the growth of $S$. enterica when paired with evolved E. coli isolates in the absence of phage. S. enterica reached an average of 1.43 -fold higher density when co-cultured with evolved T7-resistant isolates than with ancestral E. coli (Fig. 3a, $p=0.004$ ). In these co-cultures, the yield of T7resistant $E$. coli only reached $67 \%$ of the yield of the ancestor (Fig. 3a, $p=0.004$ ), suggesting that T7-resistant $E$. coli isolates supported a larger cross-feeding $S$. enterica population. We also paired P22vir-resistant $S$. enterica with ancestral E. coli and found that P22vir resistance led to an $\sim 2 \%$ decrease in $E$. coli yield (Fig. 3b, $p<0.0005$ ), and increased S. enterica 5\% (Fig. 3b, $p=0.012$ ). While our data illustrate that $S$. enteric receives more carbon from evolved E. coli than ancestral E. coli, we cannot differentiate between increased secretion or poor reuptake of carbon. These results suggest that the divergent impact of $\mathrm{T} 7$ and P22vir on community composition is in part driven by how resistance to each phage influences the abundance of cross-fed metabolites.

An alternative explanation for the asymmetric response to T7 and P22vir phage would be differences in the cost of resistance- such as decreased growth rates. However, costs

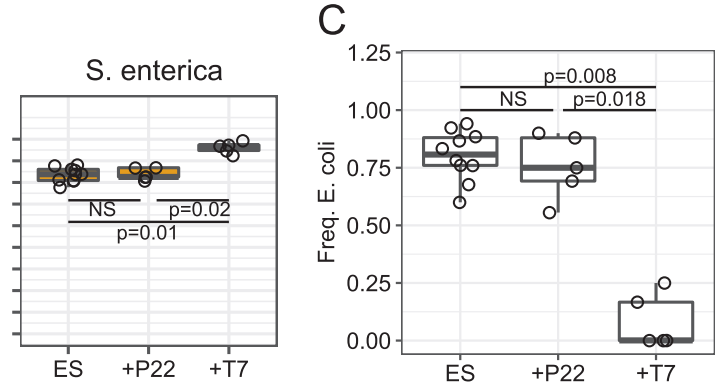

represented with frequency of E. coli at the end of growth. Ratios were calculated from plated CFUs. Statistical significance of b) and c) determined with Mann-Whitney-U test with FDR multiple hypothesis correction. $\mathrm{ES}=$ no phage controls, $+\mathrm{P} 22$ vir $($ or $+\mathrm{P} 22)=\mathrm{P} 22$ vir phage treatment, $+\mathrm{T} 7=\mathrm{T} 7$ phage treatment 

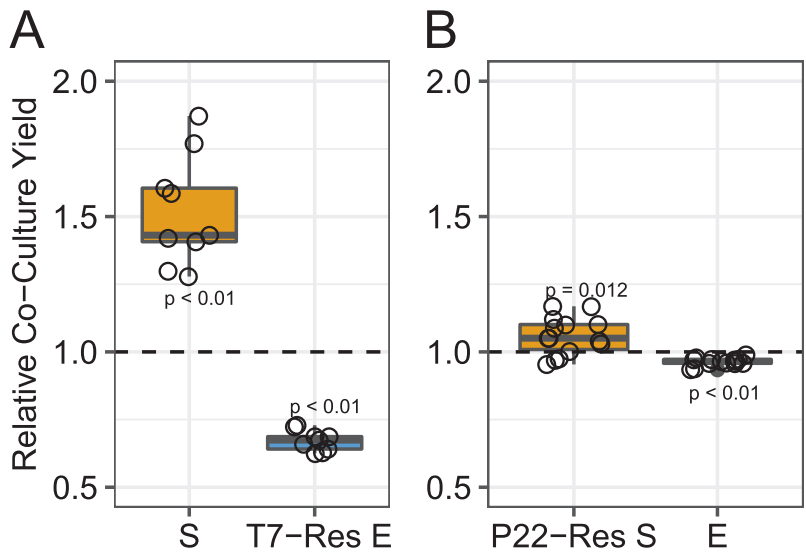

Fig. 3 Relative yield of co-cultures with resistant isolates in the absence of phage. The relative yield (CFU) for each strain in cocultures with one resistant partner as compared with the ancestral coculture. a Relative yields of co-cultured ancestral $S$. enterica (S) and T7-resistant E. coli isolates (T7-Res E) $(n=9)$. b Relative yields of cocultured P22vir-resistant S. enterica isolates (P22-Res S) and ancestral E. coli $(\mathrm{E})(n=15)$. Dashed lines represent standardized yields of ancestral $S$. enterica and E. coli co-cultures. Points represent averages of triplicate replicates. Statistical significance determined using Wilcox Sign Test with $\mu=1$

of resistance to either T7 or P22vir, measured by isolate monoculture growth rate or species-specific co-culture growth rate, were less than $8 \%$ (Supplementary Fig. 2c). Furthermore, mathematical modeling suggested that costs of resistance, encoded by decreasing growth rate when phage resistant, do not alter final yields, but caused small delays in community growth (Supplementary Fig. 2a, b).

\section{S. enterica grows on cellular debris released by phage lysis}

When lytic phage burst host cells to release phage progeny, intracellular carbon and nutrients are also released into the environment, referred to as the "viral shunt" [21]. We tested whether consumption of lysed E. coli cellular debris increased the density of $S$. enterica in the presence of $\mathrm{T} 7$ by measuring $E$. coli and $S$. enterica monoculture yields on cellular debris without phage. We produced cellular debris by sonicating monocultures of $E$. coli and $S$. enterica grown in minimal medium. We then grew ancestral $E$. coli or $S$. enterica in lysates in lactose minimal medium without methionine supplemented with $25 \%$ sonicated supernatant for $48 \mathrm{~h}$ without phage. We plated for CFUs to determine yields after $48 \mathrm{~h}$ of growth. While both $E$. coli and $S$. enterica were capable of growth in lactose minimal media supplemented with cellular debris, we observed different responses. S. enterica reached 100 -fold higher densities than $E$. coli when both were grown independently on $E$. coli cellular debris $(p=0.050)$, and two-fold higher densities than $E$. coli when both were grown independently on $S$.
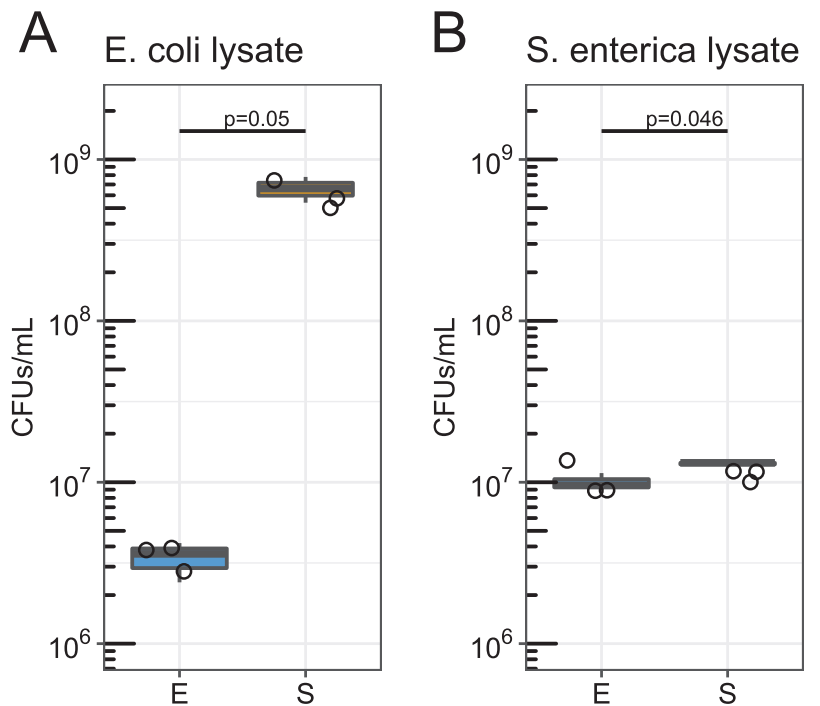

Fig. 4 Growth of S. enterica and E. coli on sonicated cellular debris. E. coli (E) and S. enterica (S) monocultures were each grown in lactose minimal medium $+25 \%$ (v/v) sonicated cellular debris lysate. a Monoculture yields on E. coli cellular debris lysate. b Monoculture yields on $S$. enterica cellular debris lysate. Cultures were inoculated with $5 \times 10^{5}$ cells $/ \mathrm{ml}$. Statistical significance was tested with a Kruskal Wallis test

enterica cellular debris ( $p=0.046$, Fig. 4). These results suggest that $S$. enterica more effeciently uses $E$. coli cellular debris released during T7 infection than $E$. coli uses $S$. enterica cellular debris released during P22vir infection. In fact, in lactose minimal medium supplemented with $25 \%$ sonicated cellular debris supernatant, $S$. enterica generated 7.3 new cells per cellular equivalent of $E$. coli cellular debris while $E$. coli generated 0.05 new cells per cellular equivalent of $S$. enterica cellular debris (Supplementary Table 4).

\section{A modified mathematical model incorporating changes in secretion profiles and cellular debris exchange does not reflect wet-lab experiments}

In wet-lab experiments, we observed that pairing T7resistant $E$. coli with ancestral $S$. enterica in cooperative coculture results in $\sim 50 \%$ more $S$. enterica than when paired with ancestral E. coli (Fig. 3). We incorporated this into our model to test how changes in secretion profile changed $S$. enterica yields. We found that the increase in acetate production by resistant $E$. coli increased simulated $S$. enterica yields 1.45 -fold compared with the base model (Fig. 5).

Wet-lab experiments also showed more efficient $S$. enterica growth on cellular debris than E. coli (Fig. 4). Therefore, we incorporated nonhost consumption of host cellular debris (cd) into our model as well. Cellular debris was included as a metabolite produced when host cells are lysed by phage (Fig. 5 and Supplementary Methods). One 
A

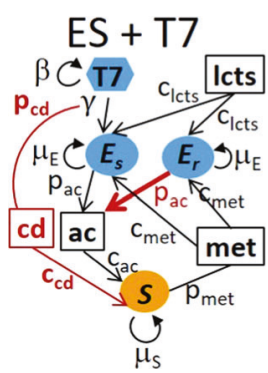

B

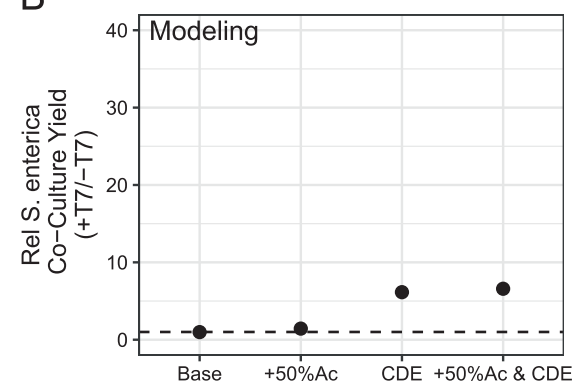

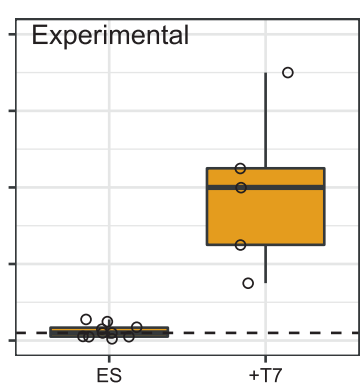

Fig. 5 Mathematical model with increased acetate production and cellular-debris exchange increases final densities of $S$. enterica during E. coli-specific T7 phage attack. a Schematic of modified model of $E$. coli-specific T7 phage attack. Red text highlights the modifications of cellular debris (cd) and a 50\% increase in acetate production $\left(\mathrm{p}_{\mathrm{ac}}\right)$ by T7-resistant E. coli. b Relative S. enterica co-culture yields of modeling (left panel) and experimental (right panel) results. Results are relative to no phage $(-\mathrm{T} 7)$ control communities. Base $=$ base model described in Fig. $1 ;+50 \% \mathrm{Ac}=\mathrm{T} 7$-resistant $E$. coli produce $50 \%$ more acetate compared with $\mathrm{T} 7$-sensitive $E$. coli; $\mathrm{CDE}=$ cellular debris exchange model where $E$. coli cells lysed by $\mathrm{T} 7$ generate cellular debris that can be used by $S$. enterica; $+50 \% \mathrm{Ac} \& \mathrm{CDE}=$ model combining increase in acetate production when $E$. coli is resistant to $\mathrm{T} 7$ phage and consumption of cellular debris by $S$. enterica lysed $E$. coli host cell was set to generate enough nutrients for $7.33 \mathrm{~S}$. enterica cells, in agreement with experimental measurements (Supplementary Table 4). Incorporating consumable cellular debris increased simulated $S$. enterica yields by 6.16-fold compared with the base model (Fig. 5).

Combining both increased acetate production and consumption of cellular debris mechanisms in the mathematical model increased the final density of $S$. enterica 6.60 -fold, still far less than that observed experimentally (Fig. 5). In fact, we had to increase the production-to-consumption ratio to $\sim 25 \mathrm{~S}$. enterica produced per lysed E. coli cell, an unrealistic number, to match the observed increases in $S$. enterica yields in wet-lab experiments (Supplementary Fig. 5). Taken together, changes in carbon secretion and consumption of cellular debris partially explain the observed increases in $S$. enterica during $\mathrm{T} 7$ infection, but additional mechanisms likely contribute.

\section{E. coli evolves partial resistance to phage, T7, increasing $S$. enterica yields}

E. coli isolates from replicate communities with $\mathrm{T} 7$ showed no inhibition of growth when cross-streaked against T7 phage (Table 1). In addition, evolved isolates grew in the presence of T7 while ancestral E. coli did not (Fig. 6b).

However, the mucoid phenotype of phage-resistant isolates suggested that partial resistance might contribute to the differential impact of $\mathrm{T} 7$ and P22vir phage on nonhost bacteria. T7 phage-resistant $E$. coli formed mucoid colonies, while P22vir-resistant $S$. enterica did not (Fig. 6a and Table 1). Mucoidy is frequently associated with partial resistance, providing incomplete protection against phage by decreasing efficiency of adsorption [26]. Finally, T7 phage increased more than ten-fold on mucoid $E$. coli isolates, much less than the 100-fold increase observed on ancestral E. coli isolates (Fig. 6c). This is qualitatively different from the full resistance observed in $S$. enterica. From an inoculum of $1 \times 10^{6} \mathrm{PFU} / \mathrm{mL}, \mathrm{P} 22$ vir phage on average reached $6.3 \times 10^{8} \pm 3 \times 10^{8} \mathrm{PFU} / \mathrm{mL}$ on ancestral $S$. enterica and $1.3 \times 10^{6} \pm 7.5 \times 10^{5} \mathrm{PFU} / \mathrm{mL}$ on $\mathrm{P} 22$ vir-resistant after $48 \mathrm{~h}$ of growth. Although growth of phage in evolved isolates may suggest $\mathrm{T} 7$ phage counter-adaptation, isolated ending phage populations and ancestral phage produced identical infection patterns when cross-streaked against evolved mucoid E. coli isolates (Supplementary Table 3). Furthermore, we note that similar phenotypes would be observed if a subset of the $E$. coli population reverted to the sensitive phenotype. However, it is unlikely that reversion instead of partial resistance would change the community-level interpretations we have suggested. These results suggest that evolved partially resistant E. coli isolates may continually be lysed throughout growth, increasing the total amount of cell debris available for $S$. enterica to consume.

Genome sequencing also supported partial resistance mechanisms in $E$. coli and a different mechanism in $S$. enterica. We whole-genome sequenced communities treated with either T7 or P22vir phage with Illumina sequencing and used breseq to identify mutations compared with reference genomes [27]. We focused on mutations unique to each phage treatment and $>20 \%$ frequency in at least one replicate community. We identified two mutations in the intergenic region of the clpx and lon genes that reach high frequency in all T7-resistant $E$. coli populations (Table 2). Mutations in the lon gene encoding a protease of $E$. coli have been shown to cause mucoid phenotypes in $E$. coli [28]. We also identified a single mutation in four of five $S$. enterica genomes of P22vir-treated communities in a Gifsy1 prophage terminase small subunit that rose to $80-90 \%$ (Table 2).

Finally, we leveraged our model to assess the impact of E. coli partial resistance to $\mathrm{T} 7$ phage on yield of the 
A

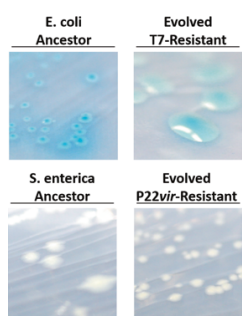

C

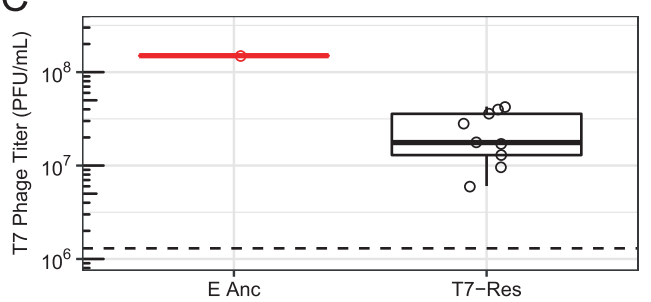

Fig. 6 Experimental partial resistance quantification of E. coli mucoid T7-resistant and implications on nonhost $S$. enterica yield during modeled T7 phage attack. a Morphology comparison of representative E. coli T7-resistant isolate or representative P22vir-resistant S. enterica isolate and ancestral strains on lactose or acetate minimal media, respectively. b Representative growth curves of ancestral E. coli (Anc $\mathrm{E}$, red line) or T7-exposed E. coli isolates (black lines) in the presence of $\mathrm{T} 7$ phage. Isolates were grown in lactose minimal medium supplemented with methionine with T7 phage ( $n=9$ isolates). c Titers of T7 phage recovered from infection of $E$. coli isolates. Isolates were inoculated at $0.01 \mathrm{OD}$ and $\sim 10^{6} \mathrm{~T} 7$ phage in lactose medium

nonhost, S. enterica. We incorporated partial resistance by decreasing the $\mathrm{T} 7$ adsorption constant to reduce the frequency of successful phage infection of partially resistant $E$. coli which results in host cells lysing and releasing cellular debris throughout growth. Adding partial resistance, in addition to increasing acetate production of T7-resistant $E$. coli and allowing $S$. enterica to consume E. coli cellular debris, led to a maximum 55.5-fold increase in S. enterica yield, which is greater than the observed wet-lab increases of S. enterica (Fig. 6d). Furthermore, intermediate adsorption values corresponding to partial resistance phenotypes led to the highest yields of S. enterica (Fig. 6d). These results suggest that multiple mechanisms including increased acetate production, consumption of phagereleased cellular debris, and partial phage-resistance mechanisms all led to the increase in the final yield of $S$. enterica during $\mathrm{T} 7$ infection of $E$. coli.

\section{Discussion}

In summary, our results suggest that lytic phage can dramatically impact communities of cooperating cross-feeding bacteria by changing yields of nonhost species through multiple mechanisms. Previously published literature about responses to abiotic stressors led us to initially predict that attack of a host species with lytic phage would suppress the
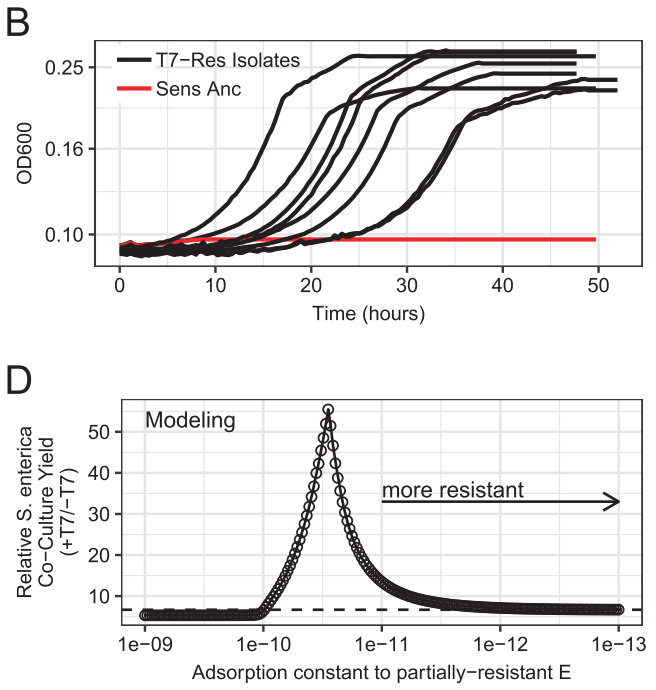

supplemented with methionine in triplicate. After culturing $72 \mathrm{~h}$ in $30^{\circ}$ C, phage lysates titered ( $n=9$ isolates, triplicate). Points are averages of three replicates. E Anc $=$ Ancestral E. coli, T7-Res Isolates = mucoid evolved T7-resistant E. coli isolates. Dashed line= starting titer. d $S$. enterica co-culture yields with varying phage adsorption constants in the resistant $E$. coli. The mathematical model used had $50 \%$ increase in acetate production when $E$. coli is resistant to T7, the ability of $S$. enterica to consume cellular debris, and partial resistance of $E$. coli against T7 phage as determined by the adsorption factor. Dashed line = Relative $S$. enterica yield in same model with full $E$. coli resistance

cross-feeding community. However, our resource-explicit models instead indicated that resistance could quickly evolve, leading to a temporary delay in community growth before ultimately reaching similar yields and species ratios to those observed in communities not attacked by phage (Fig. 1). In agreement with the mathematical model, attack of S. enterica with P22vir phage delayed community growth in wet-lab experiments and had little effect on final species ratios (Fig. 2 and Supplementary Fig. 3). In contrast, E. coli-specific $\mathrm{T} 7$ attack dramatically altered the final species ratios in favor of $S$. enterica, the nonhost, but caused a relatively small delay in community growth (Fig. 2 and Supplementary Fig. 3). Experimental and mathematical results suggest that a combination of several factors contributed to the increase of S. enterica in the presence of T7. These factors included changes in the extent of crossfeeding by resistant $E$. coli (Figs. 3 and 5), growth on cellular debris released from phage-lysed cells (Figs. 4 and 5), and the evolution of partial resistance in E. coli (Fig. 6). Our modeling suggests that divergent community responses to phage can be accurately predicted by incorporating metabolic mechanisms and the level of phage resistance.

We observed that the impact of phage extended beyond the targeted host to the other member of a cross-feeding pair. P22vir infection delayed growth of the nonhost, while T7 infection facilitated growth of the nonhost. In both cases the effect on growth of nonhosts was indirect and was 
mediated by changes in the metabolites available in the system. The P22vir results demonstrate that cross-feeding metabolic dependencies can make the entire community susceptible to perturbations of a single species, similar to previous findings with antibiotic and genetic perturbations $[18,19]$, and highlights the dangers of a cross-feeding lifestyle [29]. However, here we demonstrate that evolution of phage resistance can rapidly return cross-feeding cocultures to the unperturbed state. In contrast, $\mathrm{T} 7 \mathrm{had}$ qualitatively distinct impacts on the community that were mediated by consumption of cellular debris. Release of nutrients through cell lysis is likely to generate indirect effects on species abundance independent of microbial interactions. Indeed, viral lysis of bacteria is thought to play a major role in shaping the composition of diverse microbial communities [1, 2, 4]. Phage are frequently touted as tools for targeted treatment of pathogenic bacteria infections [7, 30]. However, our results suggest that even strainspecific phage can have broader impacts on microbial communities, which could lead to diverse phage therapy outcomes.

We predict that an understanding of what metabolites mediate cross-feeding will make predicting indirect effects of phage more accurate. We have shown two contrasting effects of phage attack on a cross-feeding microbial community. One major reason for the divergent effects is likely the identity of the cross-fed nutrients. In our microbial community, S. enterica receives acetate from E. coli, while E. coli receives methionine from S. enterica. Bennett et. al. showed that intracellular pools of carbon compounds are larger than intracellular pools of methionine [31]. Furthermore, it may be easier for cells to scavenge carbon from biomass components than methionine from proteins. Taken together, it is likely that $S$. enterica has easier access to required metabolites in cellular debris than $E$. coli because $S$. enterica reached higher densities on multiple cellular debris types than E. coli (Fig. 4 and Supplementary Table 4). In addition, the nutritional quality of cellular debris appears to vary, as the difference between $S$. enterica and $E$. coli yields on cellular debris was larger on E. coli debris than on S. enterica debris (Fig. 4 and Supplementary Table 4). We acknowledge that the metabolites released by phage lysis are likely different from those released by sonication of uninfected cells due to phage-mediated host metabolism changes [32]; however, it is unlikely that these differences would qualitatively alter our results. These results suggest that metabolic mechanisms play a critical role in determining the impact of phage in cross-feeding systems and highlight the need for further methods to quantitatively incorporate these mechanisms in our models [15].

In addition, the magnitude of the community response to T7 phage was influenced by the mechanism of phage 
resistance that evolved. Both mutations identified in E. coli genomes exposed to $\mathrm{T} 7$ phage were upstream of the lon gene encoding the lon protease, a mutation consistent with a partial resistance phenotype [28]. Down-regulation of the lon protease has been found to cause a mucoid phenotype [28] and negatively regulates the activator of capsular genes $r s c A$ [33]. Qimron et al. showed that knocking out $r s c A$ also caused mucoidy and partial phage resistance against four phage, including T7 [34]. Our model suggests that $E$. coli's incomplete resistance significantly increased the indirect effects of $E$. coli-specific T7 phage on $S$. enterica. If complete resistance was modeled, phage rapidly killed sensitive cells generating only a small pool of cellular debris. In contrast, partially resistant E. coli continued to lyse (though at a lower rate) and generate cell debris throughout growth. This continual lysis substantially increased yields of the nonhost $S$. enterica. Partial resistance should also allow the phage population to be maintained and should therefore lead to lasting changes in species ratios barring further evolution by phage or host bacteria. In conclusion, evolution of partial resistance to phage infection has the potential to generate lasting changes in community composition due to the continual generation of consumable cellular debris.

Understanding the indirect impacts of phage in microbial communities is critical as we strive to manage microbial ecosystems. Four recent studies using phage therapy in mouse models also observed extensive indirect effects of phage in microbial communities [35-38]. All four studies reported that phage therapy changed abundances of nonhost genera in mouse digestive tracts; however, no mechanisms were confirmed. Understanding how and why phage influence microbial communities is important for controlling bacteria populations, particularly in the food industry and medical field $[39,40]$. We conclude that understanding the ways that bacteria interact, the ability of species to use nutrients from lysed bacteria, and the extent of phage resistance are paramount for predicting the effects of phage attack in diverse microbial communities.

\section{Material and methods}

\section{Mathematical simulations}

We used resource-explicit ordinary differential equation models to simulate cooperative growth of $E$. coli, and $S$. enterica (Supplementary Methods). Growth of the bacterial species was governed by Monod kinetics with multiplicative limitation for resources. Production of cross-fed nutrients was growth dependent. Our base model without phage infection used two equations to directly track $E$. coli
(E) and S. enterica (S):

$$
\begin{aligned}
& \frac{d E}{d t}=E \times \mu_{E} \times \frac{1 \mathrm{cts}}{1 \mathrm{cts}+\mathrm{k}_{\mathrm{E}_{\mathrm{lcts}}}} \times \frac{\mathrm{met}}{\mathrm{met}+\mathrm{k}_{\mathrm{E}_{\mathrm{met}}}} \\
& \frac{d S}{d t}=S \times \mu_{S} \times \frac{a c}{a c+k_{S_{a c}}},
\end{aligned}
$$

where $\mathrm{E}$ or $\mathrm{S}$ is the bacterial population size, $\mu_{\mathrm{x}}$ is a speciesspecific growth rate $\left(\mathrm{h}^{-1}\right), \mathrm{k}_{\mathrm{x}}$ is a species- and metabolitespecific Monod constant, and lcts, met, and ac represent concentrations of lactose, methionine, and acetate in $\mathrm{g} /$ $200 \mu$ to compare with wet-lab results.

We added equations for $E$. coli-specific T7 or S. entericaspecific P22vir lytic phage infection (Fig. 1). For example, sensitive E. coli (Es) and $\mathrm{T} 7$ phage interacted through the following equations:

$$
\begin{aligned}
\frac{d E s}{d t}= & E s \times \mu_{E} \times \frac{1 \mathrm{lts}}{\mathrm{lcts}+\mathrm{k}_{\mathrm{E}_{\mathrm{cts}}}} \times \frac{\text { met }}{\text { met }+\mathrm{k}_{\mathrm{E}_{\mathrm{met}}}} \\
& -(E s \times T 7 \times \text { adsorption constant })
\end{aligned}
$$

$\frac{d T 7}{d t}=T 7 \times$ burst $\times E s \times$ adsorption constant

Models contain phage-sensitive $\left(\mathrm{E}_{\mathrm{s}}\right.$ or $\mathrm{S}_{\mathrm{s}}$ ) host strains, phage-resistant $\left(E_{r}\right.$ or $\left.S_{r}\right)$ host strains, or nonhost (E or $\left.S\right)$ strains as needed. Phage growth was restricted to occur only on growing cells by adding a constraint that if there were no resources, phage could not replicate (see Supplementary Methods for details). In a second model, we added an equation for cellular debris (cd) produced when sensitive host cells were killed by phage. The cellular debris was consumed by nonhost species (Fig. 5). We altered metabolite production by changing the production parameters $\left(p_{x}\right)$ and encoded partial resistance by changing the adsorption rate parameter $(\gamma)$. Our model assumes that resistant hosts were present at the beginning of the community growth and were seeded at $0.1 \%$ of the host population. All simulations were run in $\mathrm{R}$ with the DeSolve package, using the LSODA solver [41]. Refer to Supplementary Figures and Methods and Supplementary Table 1 for details.

\section{Ancestral bacterial co-culture system and viral strains}

Ancestral E. coli and S. enterica strains are previously described. Briefly, the ancestral E. coli K12 BW25113 metB::kan is a methionine auxotroph from the Keio collection with the lac operon restored. $S$. enterica LT2 was evolved to secrete methionine [25]. E. coli is tagged with a cyan fluorescent protein-encoding gene integrated at the attB lambda integration site and driven by a constitutive lambda promoter. $S$. enterica is tagged with a yellow 
fluorescent protein encoding gene under the same promoter and at the same integration site. Co-cultures were grown in lactose hypho minimal medium $(5.84 \mathrm{mM}$ lactose, $7.26 \mathrm{mM}$ $\mathrm{K}_{2} \mathrm{HPO}_{4}, 0.88 \mathrm{mM} \mathrm{NaH} \mathrm{PO}_{4}, 1.89 \mathrm{mM}\left[\mathrm{NH}_{4}\right]_{2} \mathrm{SO}_{4}, 0.41$ $\mathrm{mM} \mathrm{MgSO}_{4}$ ) [42]. Monocultures of E. coli were supplemented with $80 \mu \mathrm{M}$ of L-methionine and monocultures of $S$. enterica replaced lactose with acetate. T7 phage is an $E$. coli-specific lytic bacteriophage and P22vir phage is a $S$. enterica-specific lytic bacteriophage. Virus stocks were provided by I. J. Molineaux and were stored at $-80^{\circ} \mathrm{C}$. Working stocks of phage were grown on ancestral E. coli or $S$. enterica cultures in minimal medium and stored at $4{ }^{\circ} \mathrm{C}$.

\section{Microbial community growth}

To measure bacteria community growth, mid-log cultures started from a single bacterial colony were used to inoculate $200 \mu \mathrm{l}$ of medium in a 96-well plate with $10^{5}$ cells for each bacterial species per well, and $10^{2}$ total phage $(\mathrm{MOI}=0.01)$ where indicated (Supplementary Table 2). The 96-well plates were incubated in a Tecan Pro200 plate reader for 96-120 h at $30{ }^{\circ} \mathrm{C}$ with shaking. OD600, E. coli-specific CFP (Ex: $430 \mathrm{~nm}$; Em: $490 \mathrm{~nm}$ ), and S. enterica-specific YFP (Ex: $500 \mathrm{~nm}$; Em: $530 \mathrm{~nm}$ ) fluorescence were read every $20 \mathrm{~min}$. Fluorescent protein signals were converted to species-specific OD equivalents using an experimentallydetermined conversion factor as described [19]. Five phage treatments and five controls were used in each experiment. Phage were tested in separate experiments totaling five T7 treatments, five P22vir treatments, and 10 no-phage controls. Following growth, we plated for CFUs of both bacterial species-E. coli on lactose minimal medium plates with excess methionine and $S$. enterica on citrate minimal medium. X-gal (5-bromo-4-chloro-3-indolyl- $\beta$-D-galactopyranoside) in plates differentiated between $E$. coli and $S$. enterica. Phage population sizes were measured by plating for plaques on LB with $0.3 \%$ LB top agar with a lawn of the ancestral, sensitive host. Phage plates were incubated at $37^{\circ}$ $\mathrm{C}$ and bacterial plates at $30^{\circ} \mathrm{C}$.

\section{Testing for evolution of phage resistance: cross- streaking assays}

Three colonies per replicate community were isolated on minimal media plates. Resistance to ancestral phage was tested with cross-streaking assays on minimal media plates. Phage stock $\left(\sim 10^{8} \mathrm{PFU}\right)$ was spread in a line across a plate, bacterial isolates were streaked perpendicular to the phage culture, and plates were incubated at $30^{\circ} \mathrm{C}$ for $24-72 \mathrm{~h}$. Bacterial isolates with clearing around the phage streak were deemed sensitive and isolates with no clearing were resistant.

\section{Phenotyping phage resistant isolates}

Isolates were cultured alone or in cooperative co-culture as indicated in minimal medium in a 96-well plate. Bacteria were inoculated at $10^{5}$ cells per well. OD600, and CFP or YFP fluorescence were recorded with the TecanPro200 shaking plate reader for $72 \mathrm{~h}$. Growth rates were calculated by fitting Baranyi growth curves [43] to fluorescent protein data transformed into OD-equivalents (see above) and compared with ancestral strains grown in either monoculture or cooperative co-culture.

\section{Testing phage-mediated cellular debris exchange}

E. coli was grown in lactose + methionine minimal medium and $S$. enterica was grown in acetate minimal medium. After the stationary phase was reached $\left(\mathrm{OD}_{600} \sim 0.5\right)$, cells were pelleted, sonicated $(10,30 \mathrm{~s}$ pulses $)$, and filter sterilized with a $0.22 \mu \mathrm{m}$ filter. Filtered sonication supernatants were checked for sterility by plating. Ancestral bacteria were inoculated at $5 \times 10^{5}$ cells $/ \mathrm{ml}$ in lactose minimal medium supplemented with $25 \%$ filtered sonication supernatant and incubated at $30^{\circ} \mathrm{C}$ for $48 \mathrm{~h}$. Cultures were plated to enumerate CFUs.

\section{Whole-genome sequencing of communities and analysis}

Communities were inoculated from frozen stocks into lactose minimal media and grown at $30^{\circ} \mathrm{C}$ for 4 days. Total community DNA was isolated using the Zymo QuickgDNA Miniprep Kit (11-317C). Illumina sequencing libraries were prepared according to the Nextera XT DNA Library Prep Kit protocol, submitted to the University of Minnesota Genomics Center for QC analysis, and sequenced on an Illumina Hi-Seq with $125 \mathrm{bp}$ paired-end reads. We used the breseq tool version 0.28.1 [27] to align Illumina reads to the following reference genomes: $E$. coli str. K-12 substr. MG1655 (Accession No: NC_000913.3), S. enterica subsp. enterica serovar Typhimurium str. LT2 (Accession No: NC_003197.2, NC_003277.2), Enterobacteria phage T7 (Accession No: NC_001604.1), and Enterobacteria phage P22 (Accession No: NC_002371.2) and predict polymorphisms (-p command). Mutations lists for resistant populations were filtered to remove mutations common between ancestral strains and reference genomes. We kept mutations that were unique to each phage treatment and arose in populations $>20 \%$ (Table 2).

Acknowledgements We would like to thank Ian J. Molineaux (UTAustin) for the phage isolates, and E. Adamowicz, S. Hammarlund, and the Institute of Molecular Virology at the University of Minnesota for constructive conversations; LF was funded by a Fellowship on the 
NIH T32 AI83196 training grant and an NIH RO1 GM12149801A1 grant.

Author contributions LF and WRH designed the experiments; LF, JMC, and WRH performed mathematical modeling; LF, JA, and RHH collected and analyzed wet-lab experimental data; all authors helped write the paper.

\section{Compliance with ethical standards}

Conflict of interest The authors declare that they have no conflict of interest.

Publisher's note Springer Nature remains neutral with regard to jurisdictional claims in published maps and institutional affiliations.

Open Access This article is licensed under a Creative Commons Attribution 4.0 International License, which permits use, sharing, adaptation, distribution and reproduction in any medium or format, as long as you give appropriate credit to the original author(s) and the source, provide a link to the Creative Commons license, and indicate if changes were made. The images or other third party material in this article are included in the article's Creative Commons license, unless indicated otherwise in a credit line to the material. If material is not included in the article's Creative Commons license and your intended use is not permitted by statutory regulation or exceeds the permitted use, you will need to obtain permission directly from the copyright holder. To view a copy of this license, visit http://creativecommons. org/licenses/by/4.0/.

\section{References}

1. Sullivan MB, Weitz JS, Wilhelm S. Viral ecology comes of age. Environ Microbiol Rep 2017;9:33-5.

2. Suttle CA. Marine viruses-major players in the global ecosystem. Nat Rev Microbiol 2007;5:801-12.

3. Rosenwasser S, Ziv C, Creveld SG, van, Vardi A. Virocell metabolism: metabolic innovations during host-virus interactions in the ocean. Trends Microbiol 2016;24:821-32.

4. Breitbart M, Bonnain C, Malki K, Sawaya NA. Phage puppet masters of the marine microbial realm. Nat Microbiol 2018;3: 754-66.

5. Santos SB, Costa AR, Carvalho C, Nóbrega FL, Azeredo J. Exploiting bacteriophage proteomes: the hidden biotechnological potential. Trends Biotechnol 2018;36:966-84.

6. de Melo AG, Levesque S, Moineau S. Phages as friends and enemies in food processing. Curr Opin Biotechnol 2018;49: 185-90.

7. Kakasis A, Panitsa G. Bacteriophage therapy as an alternative treatment for human infections: a comprehensive review. Int $\mathbf{J}$ Antimicrob Agents 2019;53:16-21.

8. Scanlan PD. Bacteria-bacteriophage coevolution in the human gut: implications for microbial diversity and functionality. Trends Microbiol 2017;25:614-23.

9. Harcombe WR, Bull JJ. Impact of phages on two-species bacterial communities. Appl Environ Microbiol 2005;71:5254-9.

10. Brockhurst MA, Fenton A, Roulston B, Rainey PB. The impact of phages on interspecific competition in experimental populations of bacteria. BMC Ecol 2006;6:19.

11. Yu P, Mathieu J, Yang Y, Alvarez PJJ. Suppression of enteric bacteria by bacteriophages: importance of phage polyvalence in the presence of soil bacteria. Environ Sci Technol 2017;51:5270-8.
12. Maslov S, Sneppen K. Population cycles and species diversity in dynamic Kill-the-Winner model of microbial ecosystems. Sci Rep 2017;7:39642.

13. Fahimipour AK, Anderson KE, Williams RJ. Compensation masks trophic cascades in complex food webs. Theor Ecol 2017;10:245-53.

14. D’Souza G, Shitut S, Preussger D, Yousif G, Waschina S, Kost C. Ecology and evolution of metabolic cross-feeding interactions in bacteria. Nat Prod Rep. 2018;35:455-88.

15. Carlson RP, Beck AE, Phalak P, Fields MW, Gedeon T, Hanley $\mathrm{L}$, et al. Competitive resource allocation to metabolic pathways contributes to overflow metabolisms and emergent properties in cross-feeding microbial consortia. Biochem Soc Trans 2018;46: 269-84.

16. Shou W, Ram S, Vilar JMG. Synthetic cooperation in engineered yeast populations. PNAS 2007;104:1877-82.

17. Harcombe WR, Riehl WJ, Dukovski I, Granger BR, Betts A, Lang $\mathrm{AH}$, et al. Metabolic resource allocation in individual microbes determines ecosystem interactions and spatial dynamics. Cell Rep 2014;7:1104-15.

18. Chubiz LM, Harcombe WR, Granger BR, Segrè D. Species interactions differ in their genetic robustness. Front Microbiol 2015;6:271.

19. Adamowicz EM, Flynn J, Hunter RC, Harcombe WR. Crossfeeding modulates antibiotic tolerance in bacterial communities. ISME J 2018;12:2723-35.

20. Haraldsson M, Gerphagnon MM, Bazin P, Colombet J, Tecchio S, Sime-Ngando TT, et al. Microbial parasites make cyanobacteria blooms less of a trophic dead end than commonly assumed. ISME J 2018;12:1008-20.

21. Wilhelm SW, Suttle CA. Viruses and nutrient cycles in the sea: viruses play critical roles in the structure and function of aquatic food webs. Bioscience 1999;49:781-8.

22. Weitz JS, Stock CA, Wilhelm SW, Bourouiba L, Coleman ML, Buchan A, et al. A multitrophic model to quantify the effects of marine viruses on microbial food webs and ecosystem processes. ISME J 2015;9:1352-64.

23. Hammarlund SP, Chacón JM, Harcombe WR. A shared limiting resource leads to competitive exclusion in a cross-feeding system. Environ Microbiol. 2018; https://doi.org/10.1111/1462-2920. 14493.

24. Hoek TA, Axelrod K, Biancalani T, Yurtsev EA, Liu J, Gore J. Resource availability modulates the cooperative and competitive nature of a microbial cross-feeding mutualism. PLOS Biol 2016;14:e1002540.

25. Harcombe WR. Novel cooperation experimentally evolved between species. Evolution 2010;64:2166-72.

26. Scanlan PD, Buckling A. Co-evolution with lytic phage selects for the mucoid phenotype of Pseudomonas fluorescens SBW25. ISME J 2012;6:1148-58

27. Deatherage DE, Barrick JE. Identification of mutations in laboratory evolved microbes from next-generation sequencing data using breseq. Methods Mol Biol 2014;1151:165-88.

28. Torres-Cabassa AS, Gottesman S. Capsule synthesis in Escherichia coli K-12 is regulated by proteolysis. J Bacteriol 1987;169: 981-9.

29. Oliveira NM, Niehus R, Foster KR. Evolutionary limits to cooperation in microbial communities. PNAS 2014;111:17941-6.

30. Levin BR, Bull JJ. Population and evolutionary dynamics of phage therapy. Nat Rev Microbiol 2004;2:166-73.

31. Bennett BD, Kimball EH, Gao M, Osterhout R, Van Dien SJ, Rabinowitz JD. Absolute metabolite concentrations and implied enzyme active site occupancy in Escherichia coli. Nat Chem Biol 2009;5:593-9. 
32. Birch EW, Ruggero NA, Covert MW. Determining host metabolic limitations on viral replication via integrated modeling and experimental perturbation. Plos Comput Biol 2012;8:e1002746.

33. Gottesman S, Trisler P, Torres-Cabassa AS. Regulation of capsular polysaccharide synthesis in Escherichia coli K-12: Characterization of three regulatory genes. J Bacteriol 1985;162:1111-9.

34. Qimron U, Marintcheva B, Tabor S, Richardson CC. Genomewide screens for Escherichia coli genes affecting growth of T7 bacteriophage. PNAS 2006;103:19039-44.

35. Hsu BB, Gibson TE, Yeliseyev V, Liu Q, Lyon L, Bry L, et al. Dynamic Modulation of the Gut Microbiota and Metabolome by Bacteriophages in a Mouse Model. Cell Host Microbe 2019;25:803-14.e5.

36. Galtier M, De Sordi L, Maura D, Arachchi H, Volant S, Dillies $\mathrm{M}-\mathrm{A}$, et al. Bacteriophages to reduce gut carriage of antibiotic resistant uropathogens with low impact on microbiota composition. Environ Microbiol 2016;18:2237-45.

37. Mai V, Ukhanova M, Reinhard MK, Li M, Sulakvelidze A, Alexander, et al. Bacteriophage administration significantly reduces Shigella colonization and shedding by Shigella-challenged mice without deleterious side effects and distortions in the gut microbiota. Bacteriophage 2015;5:e1088124.

38. Reyes A, Wu M, McNulty NP, Rohwer FL, Gordon JI. Gnotobiotic mouse model of phage-bacterial host dynamics in the human gut. PNAS. 2013;110:20236-41.

39. Szafrański SP, Winkel A, Stiesch M. The use of bacteriophages to biocontrol oral biofilms. J Biotechnol 2017;250:29-44.

40. Baker JL, Edlund A. Exploiting the oral microbiome to prevent tooth decay: Has evolution already provided the best tools? Front Microbiol 2018;9:3323.

41. Soetaert K, Petzoldt T, Setzer RW Solving differential equations in R: Package deSolve. J Stat Softw. 2010;33.

42. Delaney NF, Kaczmarek ME, Ward LM, Swanson PK, Lee M-C, Marx CJ. Development of an optimized medium, strain and highthroughput culturing methods for Methylobacterium extorquens. PLoS One 2013;8:e62957.

43. Baranyi J, Roberts TA. A dynamic approach to predicting bacterial growth in food. Int J Food Microbiol 1994;23:277-94. 